\title{
Instituto de Investigaciones de la Altura: Buscando la solución a problemas nacionales
}

\author{
Altitude Research Institute: \\ Looking for the solution to national problems
}

\author{
Roberto Alfonso-Accinelli ${ }^{1,2,3}$, Cinthya Vásquez-Velásquez ${ }^{3,4}$, Diego Fano-Sizgorich ${ }^{3,4}$, \\ José Luis Macarlupu ${ }^{3,5}$, Gustavo Francisco Gonzales ${ }^{3,4}$, José Enrique Viton-Rubio ${ }^{1,3}$
}

\section{Resumen}

El Instituto de Investigaciones de Altura (IIA) nace hace 60 años con la creación de la Universidad Cayetano Heredia (UPCH). Está orientado a la investigación de la adaptación y aclimatación a la altura, y sin pretenderlo a la solución de los problemas nacionales en salud. La erradicación del bocio endémico, la consolidación del Programa Piloto de Tuberculosis y su expansión a un modelo comunitario, el plantear que la anemia en el Perú es por sobrediagnóstico al usar una inadecuada fórmula de corrección por la altura y un punto de corte plano para los niños, el conocer el efecto beneficioso de plantas originarias como la maca, el tratamiento para el COVID-19 con medicamentos en segunda indicación, el daño a la salud por el uso de cocinas tradicionales de biomasa son algunos de los problemas cuyas soluciones han nacido de sus investigaciones, además de las encontradas por sus investigaciones sobre los problemas de la altura.

Palabras clave: Altura, anemia, COVID-19, combustibles sólidos.

\section{Abstract}

The Instituto de Investigaciones de la Altura (IIA) was born 60 years ago with the creation of the Cayetano Heredia University (UPCH).It is oriented to the investigation of the adaptation and acclimatization to the altitude, and without pretending it to the solution of the national problems in health. The eradication of endemic goiter, the consolidation of the Tuberculosis Pilot Program and its expansion to a community model, the proposition that anemia in Peru is due to overdiagnosis by using an inadequate correction formula for height and a flat cut-off point for the children, knowing the beneficial effect of native plants such as maca, treatment for COVID-19 with second-indication drugs, damage to health from the use of traditional biomass stoves are some of the problems whose solutions have been born from his investigations, in addition to those found by his investigations on the problems of height.

Keywords: Altitude, anemia, COVID-19, solidfuels (source: MeSHNLM).

Desde su nacimiento, cuando se fundó la Universidad Peruana Cayetano Heredia, el Instituto de Investigaciones de la Altura (IIA) se ha orientado a la investigación de la adaptación y aclimatación a la altura y sin pretenderlo a la solución de los problemas nacionales en salud. La erradicación del bocio endémico por el Dr. Eduardo Pretell Zárate ${ }^{(1,2)}$, la consolidación del Programa Piloto de Tuberculosis por el Dr. Amador
Carcelén Bustamante y su expansión a un modelo comunitario en los centros de salud por el Dr. Roberto Accinelli Tanaka, son un ejemplo de modelos de intervención en salud logrados desde el IIA ${ }^{(3)}$.

El inicio de la tercera década del siglo XXI lo recordaremos por la aparición de la pandemia que está

${ }^{1}$ Facultad de Medicina Alberto Hurtado, Universidad Peruana Cayetano Heredia, Lima, Perú. ${ }^{2}$ Servicio de Neumología, Hospital Cayetano Heredia, Lima, Perú. 'Instituto de Investigaciones de la Altura. Universidad Peruana Cayetano Heredia, Lima, Perú. ${ }^{4}$ Laboratorio de Endocrinología y Reproducción, Departamento de Ciencias Biológicas y Fisiológicas, Facultad de Ciencias y Filosofía, Universidad Peruana Cayetano Heredia. Lima, Perú. Laboratorio de Fisiología Comparada, Departamento de Ciencias Biológicas y Fisiológicas, Facultad de Ciencias y Filosofí, Universidad Peruana Cayetano Heredia. Lima, Perú. 
cambiando al mundo. Ante la enfermedad por coronavirus 2019 (COVID19) los integrantes del IIA respondieron con una publicación que sintetizaba lo conocido hasta el momento sobre el coronavirus tipo 2, causante del síndrome respiratorio agudo severo (SARS-CoV-2) y cómo combatirlo ${ }^{(4)}$. El uso de las medicinas en segunda indicación, hidroxicloroquina y cloroquina, consideradas en el 2006 como el tratamiento para la reaparición del SARS o de un nuevo coronavirus, fue considerado luego de los estudios en humanos el 2020 como el tratamiento de elección ${ }^{(4)}$. La hidroxicloroquina con azitromicina disminuyó en Tahuantinsuyo Bajo en 6.5 veces la mortalidad en pacientes en fase viral comparada con la cohorte nacional peruana ${ }^{(5)}$.

Otra alternativa es la ivermectina, usada en el Perú cuando no hubo disponibilidad del uso de antimaláricos, que llevó a los miembros del IIA a hacer una de las primeras revisiones publicadas sobre la utilidad de este medicamento en COVID-19 $9^{(6)}$. Hoy, en que la mortalidad por COVID-19 amenaza con seguir incontrolable ${ }^{(7,8)}$, los miembros del Laboratorio de Respiración afirman que usando la ivermectina como tratamiento preventivo pre y post-exposición al SARS$\mathrm{CoV}-2$ y la hidroxicloroquina con azitromicina para el de los enfermos, se lograría controlar la pandemia en un mes en el Perú.

Desde abril del $2019^{(9)}$ semanalmente ha habido reuniones de coordinación y de capacitación con los equipos de salud de las 6 regiones que componen la Mancomunidad de los Andes con una participación por ZOOM siempre al tope de 500 personas y por Facebook hasta más de 200,000, habiendo ingresado a verlas hasta más de $500,000^{(10,11)}$. Compartir conocimientos, resultados de los tratamientos y de las intervenciones en COVID-19, pero principalmente esperanza en que se puede controlar la pandemia, han sido las grandes motivaciones que han logrado la masiva participación en estas reuniones.

El grupo latinoamericano, incluido el IIA, ha realizado investigaciones que demuestran que en la altura hay una menor frecuencia y mortalidad por COVID-19 $9^{(12-14)}$. Solís y Zubieta postularon que era por la menor biodisponibilidad de receptores ECA-2, y el grupo del IIA explica que es la misma causa de la menor mortalidad en mujeres.

Anemia en niños es un problema nacional y mundial sin solución. En el Perú, un Programa Nacional que no disminuye en casi una década la tasa de alrededor del $43 \%$, cuando en la misma población la tasa de desnutrición crónica ha bajado a la mitad, nos lleva a plantear el porqué de esta diferencia ${ }^{(15)}$. Los trabajos del Dr. Gustavo Gonzales nos dan la respuesta. Hace 9 años, Gonzales fue el primero en el mundo que llamó la atención sobre el inadecuado factor de corrección usado para determinar los puntos de corte para el diagnóstico de anemia, por lo que se sobreestima la misma entre los pobladores de la altura $^{(16)}$. Él y su grupo han seguido con sus investigaciones y, sumadas a las de Accinelli, se ha visto que el sobrediagnóstico, por una inadecuada ecuación de corrección por la altura de residencia y por usar la OMS un punto de corte plano y único para los menores de 5 años, sería la explicación ${ }^{(17)}$.

En los niños peruanos menores de tres años Accinelli determinó que los valores de anemia dependen de la edad y de la altura de residencia y, que con un nomograma que relacione estos factores, se puede conocer fácilmente qué niño es anémico, eliminándose así el actual sobrediagnóstico ${ }^{(17)}$. En el Taller Addressing the challenges of anaemia in the Andean Region, realizado el 20 y 21 de enero del 2021, coorganizado por la Academia de Ciencias Médicas del Reino Unido y la Academia de Ciencias del Perú, con la participación de los expertos mundiales en el tema, se dio por fin un apoyo a lo propuesto por Gonzales del IIA, pues se concluyó que la anemia está siendo sobre-diagnosticada en la altura... los ajustes de los umbrales conducen al diagnóstico de anemia en muchos niños con niveles suficientes de hierro y hemoglobina. Esto podría llevar a un tratamiento excesivo... y a la necesidad de programas de suplementación con hierro ${ }^{(18)}$.

El valor de la hemoglobina depende de los niveles de eritropoyetina, hormona renal que se incrementa con la hipoxemia, para así mantener el contenido de oxígeno que llega a los tejidos. Villafuerte ha encontrado que en la altura la eritrocitosis excesiva se relaciona también con los niveles del factor soluble de la eritropoyetina y más con la relación entre eritropoyetina/receptor soluble de eritropoyetina ${ }^{(19)}$. Sería entonces la eritropoyetina libre la que determina la eritrocitosis excesiva. Finalmente, ha encontrado que en condiciones de hipoxia en personas con mal de montaña crónico (MMC) las células progenitoras a nivel de los precursores de los eritrocitos en personas con este mal producen una mayor proporción de precursores eritroides y que SENP1 es esencial para la proliferación ${ }^{(19)}$. La eritrocitosis excesiva dependería además de factores genéticos que actuarían a nivel de la médula ósea elevando finalmente los glóbulos rojos circulantes.

Veinte años viene estudiándose por el IIA la maca (Lepidium meyenii), mostrando que la maca negra incrementa la función testicular en ratas ${ }^{(20)}$. Tiene un efecto significativo sobre la resistencia física, la regulación del estrés, función 
cognitiva y el $\mathrm{MMC}^{(21,22)}$. Su aplicación tópica acelera el proceso de cicatrización, además de evitar la formación de cicatrices hipertróficas o queloides, y actúa como un potente inmunomodulador, favoreciendo la expresión de citoquinas antiinflamatorias como el interferón-gamma ${ }^{(23)}$. Este último efecto, sumado a rol modulador de los receptores de estrógenos, también contribuye en la reducción del tamaño de la próstata en modelos de hiperplasia prostática benigna ${ }^{(24,25)}$. Se ha tratado de cultivar en otros países, aunque sin mayor éxito, por lo que el impacto de estas investigaciones ha hecho que la maca se revalorice, llevando a la explosión productiva y de exportación de la maca, generando una bonanza económica en las localidades productoras de la meseta del Bombón en Junín.

El Laboratorio de Endocrinología y Reproducción ha llevado a cabo un macro proyecto de investigación enfocado en salud ambiental, el Regional GEOHealth Hub Centered in Peru. Han hallado que $66 \%$ de la población tacneña se encuentra expuesta a niveles de arsénico por encima del límite de $10 \mu \mathrm{g} / \mathrm{L}^{(26)}$ lo que es un riesgo para la salud especialmente en poblaciones vulnerables como las gestantes ${ }^{(27,28)}$.

Accinelli y León-Abarca ${ }^{(29)}$ encontraron que en los países que tienen un mayor uso de combustibles sólidos (biomasa y carbón) sus niños presentaban mayor frecuencia de infecciones respiratorias agudas (IRAS) y de anemia. Así, la contaminación intradomiciliaria sería la explicación de por qué los pobres fallecen más por IRAS y al causar también una mayor inflamación estos niños tendrían anemia por el incremento de IL-6 que provoca elevación de la producción de hepcidina. Estos estudios obligan a buscar soluciones inmediatas y sostenibles al uso de las cocinas tradicionales de biomasa que serían el factor prevenible de mayor impacto a la salud global.

El Laboratorio de Endocrinología y Reproducción en cooperación con el Ministerio de Salud, hospitales y distintas Direcciones Regionales de Salud ha evaluado el impacto de políticas públicas sobre la salud, mereciendo destacar el efecto del Plan Pico y Placa en la disminución de la contaminación aérea en Lima, observándose una considerable reducción del PM2.5 en la Av. Abancay ${ }^{(30)}$.

El MMC lo presenta el que tiene eritrocitosis excesiva junto con una puntuación de síntomas mayor de 5 , y está presente en el $15 \%$ de los residentes de altura. Macarlupú ${ }^{(31)}$ acaba de demostrar que el ejercicio aeróbico submáximo disminuyó el hematocrito en un $5 \%$ y un $7 \%(\mathrm{p}<0.05)$ a la semana 4 y 8 , respectivamente, mientras que la puntuación de MMC también se redujo en $4.8 \pm 1.3$ y $3.5 \pm 0.8$, respectivamente $(\mathrm{p}<0.05)$. Halló una relación inversa entre hematocrito y haptoglobina, lo que indicaría como probable mecanismo hemólisis por el ejercicio. Tendríamos un tratamiento sencillo, con lo que se evitaría recomendar el actual, que consiste en hacer descender a alturas más bajas al enfermo con MMC, que no todos los siguen porque no desean abandonar su lugar de residencia.

En cuanto a la adaptación aguda a la altura, Ju y Zhang ${ }^{(32)}$ del Laboratorio de Respiración, encontraron en personas jóvenes sanas que viajaron a Ayacucho (2761 msnm), que habría dos fenotipos: Uno, con saturación de oxígeno nocturna media baja al recién llegar, que aumentó conforme fueron pasando los días de residencia en la altura, y otro con saturación en niveles dentro de lo esperado para esa altura. Los primeros tenían el índice ápnea-hipopnea (IAH) más bajo que los segundos. Esto sugiere dos fenotipos distintos: O respiras pero no duermes o duermes pero no respiras. Se necesitan continuar estudios con poblaciones más grandes y a mayores alturas para poder ver su relación con el mal de montaña agudo.

Han pasado 60 años de la fundación de la UPCH y del IIA. Los trabajos realizados en el último quinquenio confirman que la investigación es parte de la tradición no perdida a pesar de las décadas transcurridas. Y que un país pobre como el nuestro ahorra recursos inmensos al presentar respuestas sencillas a los problemas diarios de salud. Esperemos que en los próximos sesenta años la tradición persista y que la UPCH y el IIA sigan investigando y buscando respuestas a nuestros problemas.

\section{Referencias bibliográficas}

1.Pretell EA, Pearce EN, Moreno SA, et al. Elimination of iodine deficiency disorders from the Americas: a public health triumph. Lancet Diabetes Endocrinol. 2017;(6):412-414.

2.Pretell EA, Delange F, Hostalek U, et al. Iodine nutrition improves in Latin America. Thyroid. 2004;14(8):590-599.

3.Carcelén A, Ruiz R, Accinelli RA, et al. Evaluación del Programa de Tuberculosis del Hospital General Base Cayetano Heredia. XIV
Congreso Nacional de tuberculosis y enfermedades del tórax 1981. Libro de Resúmenes: pág. 189.

4. Accinelli RA, Zhang CM, Ju JD, et al. COVID-19: la pandemiapor el nuevo virus SARS-CoV-2. Rev Peru Med Exp Salud Pública. 2020 Apr-Jun 2020;37(2):302-311.

5. Accinelli RA, Ynga GJ, Leon JA, et al. Hydroxychloroquine / azithromycin in COVID-19: The association between time to 
treatment and case fatality rate. Travel Med Infect Dis. 2021 Sep $14 ; 44: 102163$

6. Portmann A, Bryce M, Accinelli RA. Antiviral and AntiInflammatory Properties of Ivermectin and Its Potential Use in COVID-19. Arch Bronconeumol.2020;56(12):831.

7.COVID-19 Coronavirus Pandemic. Worldometers.info (Obtenida Mayo 14,2021, en https://www.worldometers.info/coro navirus $/ \% 0 \mathrm{~A}$ )

8. Sala Situacional COVID-19 Perú. Ministerio de Salud del Perú.2021. (Obtenida mayo 14,2021, en https://covid19.minsa.gob .pe/sala_situacional.asp)

9. Acciones y/o Proyectos de Salud 2020. Mancomunidad Regional de los Andes. (Obtenida mayo 14,2021, en https://www.mancomu nidadwaewgionaldelosandes.gob.pe/index.php/articula cionterritorial/comis ion-de-salud/comision-de-salud-2/comisiondesalud-8)

10. Experiencias en el manejo de pacientes con COVID-19. Mancomunidad Regional de los Andes. (Obtenida mayo 14,2021, en https://www.mancomunidadregionaldelosandes. gob.pe/index.php/component/k2/item/41-experiencias-en-elmanejo-depacientes-con-covid-19)

11. Experiencias contadas por personas que superaron la COVID19. Mancomunidad Regional de los Andes. Yo me curé del COVID-19 (Obtenida mayo 14,2021, en https://www.mancomuni dadregionaldelosandes.gob.pe/index.php/component/k2/item/41experiencias-en-el-manejo-de-pacientes-con-covid-19)

12. Arias C, Carvajal F, Poma $L$, et al. Decreased incidence, virus transmission capacity, and severity of COVID-19 at altitude on the American continent. PLoS One. 2021;16(3):e0237294.

13. Accinelli RA, Leon JA. Lower frequency and letality in women and in altitude due to COVID-19: Two sides of the same coin. Arch Bronconeumol. 2021;57 Suppl 2:70-72.

14. Accinelli RA, Leon JA. At High Altitude COVID-19 Is Less Frequent: The Experience of Peru. Arch Bronconeumol. 2020 ;56(11):760-761.

15. Accinelli RA, Gonzales G, Ruiz W, et al. Informe sobre la situación de la anemia en el Perú. Diagnóstico. 2020;57(3):157-159.

16. Gonzales GF. Diferencias en la detección de anemia en la altura según la Organización Mundial de la Salud - Réplica de autores. Rev Peru Med Salud Pública; 2012;29(1):158-159.

17. Accinelli RA, Leon JA. Age and altitude of residence determine anemia prevalence in Peruvian 6 to 35 months old children. PLoS One. 2020;15(1):e0226846-e0226846.

18. Addressing the challenges of anaemia in the Andean Region. The Academy of Medical Science. Webinar (Obtenida mayo 14,2021, en https://acmedsci.ac.uk/more/events/webinar:addressing-the-challenges-of-anaemia-in-the-andean-region)

19. Villafuerte FC, Macarlupú JL, Anza-Ramírez C, et al. Decreased plasma soluble erythropoietin receptor in high-altitude excessive erythrocytosis and Chronic Mountain Sickness. J Appl
Physiol. 2014;117(11):1356-1362.

20.Gonzales G, Rubio J, Chung A, et al. Effect of alcoholic extract of Lepidium meyenii (Maca) on testicular function in male rats. Asian J Androl. 2003 Dec;5(4):349-352.

21. Gonzales C, Yupanqui I, Montero E, et al. Acceptability, safety, and efficacy of oral administration of extracts of black or red maca (Lepidium meyenii) in adult human subjects: A randomized, double-blind, placebo-controlled study. Pharmaceuticals. 2016 Aug 18;9(3):49.

22.Gonzales G, Villaorduña L, Gasco M, et al. Maca (Lepidium Meyenii Walp), a review of its biological properties. Rev Peru Med Exp Salud Pública 2014;31(1):100-110.

23.Nuñez $D$, Olavegoya $P$, Gonzales $G$, et al. Red Maca (Lepidiummeyenii), a Plant from the Peruvian Highlands, Promotes Skin Wound Healing at Sea Level and at High Altitude in Adult Male Mice. High Alt Med Biol. 2017 Dec;18(4):372-383.

24.Fano D, Vásquez C, Gonzales $C$, et al. N-butanol and aqueous fractions of red maca methanolic extract exerts opposite effects on androgen and oestrogens receptors (alpha and beta) in rats with testosterone-induced benign prostatic hyperplasia. EvidenceBased Complementary and Alternative Medicine, 2017.

25.Vásquez $\mathbf{C}$, Gasco $M$, Fano $D$, et al. Inflammatory pathway employed by Red Maca to treat induced benign prostatic hyperplasia in rats. Andrologia. 2020;52(3):e13516.

26. Fano D, Vásquez $\mathbf{C}$, Aguilar $\mathbf{J}$, et al. Arsenic Concentrations in Household Drinking Water: A Cross-Sectional Survey of Pregnant Women in Tacna, Peru, 2019. Expo Health. 2020;12(4):555-560.

27. Fano D, Vásquez C, Ramirez C, et al. Reproductive outcomes in pregnant women and its association with arsenic contamination in drinking water, in a region characterized by high birth weight rates in Peru. J Matern Fetal Neonatal Med. 2019 Dec; 16:1-3.

28. Fano D, Vásquez $\mathbf{C}$, Yucra $\mathbf{S}$, et al. Total Urinary Arsenic and Inorganic Arsenic Concentrations and Birth Outcomes in Pregnant Women of Tacna, Peru: A Cross-Sectional Study. Expo Health. 2021 Mar; 13(1):133-140.

29. Accinelli RA, Leon JA, Gozal D. Ecological study on solid fuel use and pneumonia in young children: A worldwide association. Respirology. 2017;22(1):149-156.

30.Tapia V, Carbajal L, Vásquez V, et al. Traffic regulation and environmental pollution by particulate material (2.5 and 10), sulfur dioxide, and nitrogen dioxide in Metropolitan Lima, Peru. Rev Peru Med Exp Salud Publica. 2018 Apr-Jun; 35(2):190-197.

31. Marcalupú JL, Vizcardo G, Figueroa R, et al. Short-term Submaximal Aerobic Training Reduces Hematocrit and SymptomatoSymptomatology in Andean Highlanders with Monge's Disease. FASEB J. 2020;34(1):1-1.

32.Jia J, Zhang C, Sgambati FP, et al. Acute Altitude Acclimatiza tion in Young Healthy Volunteers: Nocturnal Oxygenation Increases Over Time, Whereas Periodic Breathing Persists. High Alt Med Biol. 2021 Mar;22(1):14-23.

Contribución de autoría: Roberto Alfonso Accinelli ha contribuido con la conceptualización del artículo. Todos los autores contribuyeron en la recolección de información, redacción y aprobación de la versión final. Conflicto de interés: Los autores no tienen conflictos de interés con la publicación de este trabajo.

Financiamiento: Autofinanciado

Citar como: Accinelli RA., Vásquez-Velásquez C., Fano-Sizgorich D., Macarlupú JL., Gonzales GF, VitonRubio JE. Instituto de Investigaciones de la Altura: Buscando la solución a problemas nacionales. Diagnóstico(Lima). 2021;60(3):166-169.

DOI: 10.33734 /diagnostico.v60i3.306

Correspondencia: Roberto Alfonso Accinelli. Correo electrónico: roberto.accinelli@upch.pe 